\title{
BMJ Open Association between probable postnatal depression and increased infant mortality and morbidity: findings from the DON population-based cohort study in rural Ghana
}

Benedict Weobong, ${ }^{1,2}$ Augustinus H A ten Asbroek, ${ }^{2,3}$ Seyi Soremekun, ${ }^{2}$ Lu Gram, ${ }^{2}$ Seeba Amenga-Etego, ${ }^{1}$ Samuel Danso, ${ }^{1}$ Seth Owusu-Agyei, ${ }^{1,2}$ Martin Prince, ${ }^{4}$ Betty R Kirkwood ${ }^{2}$

To cite: Weobong $B$, ten Asbroek AHA, Soremekun S, et al. Association between probable postnatal depression and increased infant mortality and morbidity: findings from the DON population-based cohort study in rural Ghana. BMJ Open 2015;5:e006509. doi:10.1136/bmjopen-2014006509

- Prepublication history and additional material is available. To view please visit the journal (http://dx.doi.org/ 10.1136/bmjopen-2014006509).

MP and BRK are joint last authors.

Received 1 September 2014 Revised 13 February 2015 Accepted 28 February 2015

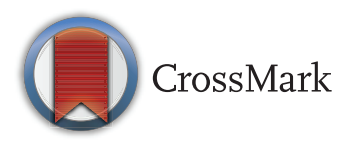

For numbered affiliations see end of article.

Correspondence to Dr Benedict Weobong; bkweobong@gmail.com

\section{ABSTRACT}

Objectives: To assess the impact of probable depression in the immediate postnatal period on subsequent infant mortality and morbidity.

Design: Cohort study nested within 4 weekly surveillance of all women of reproductive age to identify pregnancies and collect data on births and deaths.

Setting: Rural/periurban communities within the Kintampo Health Research Centre study area of the Brong-Ahafo Region of Ghana.

Participants: 16560 mothers who had a live singleton birth reported between 24 March 2008 and 11 July 2009, who were screened for probable postnatal depression ( $p P N D$ ) between 4 and 12 weeks post partum (some of whom had also had depression assessed at pregnancy), and whose infants survived to this point.

Primary/secondary outcome measures: All-cause early infant mortality expressed per 1000 infant-months of follow-up from the time of postnatal assessment to 6 months of age. The secondary outcomes were (1) all-cause infant mortality from the time of postnatal assessment to 12 months of age and (2) reported infant morbidity from the time of the postnatal assessment to 12 months of age.

Results: 130 infant deaths were recorded and singletons were followed for 67457.4 infant-months from the time of their mothers' postnatal depression assessment. pPND was associated with an almost threefold increased risk of mortality up to 6 months (adjusted rate ratio (RR), 2.86 (1.58 to 5.19); $p=0.001$ ). The RR up to 12 months was 1.88 (1.09 to 3.24; $p=0.023)$. pPND was also associated with increased risk of infant morbidity.

Conclusions: There is new evidence for the association between maternal pPND and infant mortality in low-income and middle-income countries. Implementation of the WHO's Mental Health Gap Action Programme (mhGAP) to scale up packages of care integrated with maternal health is encouraged as an important adjunct to child survival efforts.

\section{Strengths and limitations of this study}

- Large prospective cohort.

- Focus on depression over the immediate postnatal period, and on infant mortality in the first 6 months of life.

- Strong supporting evidence of independent associations with infant morbidity that provides some evidence of the likely pathways leading to early infant death.

- Cannot exclude respondent bias, particularly an effect of maternal depression on the mothers' reports of infant morbidity.

- Establishing causality is challenging, given the often chronic, remitting and relapsing nature of depression.

\section{INTRODUCTION}

Maternal depression, a common and debilitating mental disorder, is known to be associated with adverse effects on infant and child health and development. ${ }^{1-3}$ While there is clear evidence of increased risk of stillbirth, and suggestive evidence of increased mortality among offspring of mothers with serious mental illnesses (psychotic disorders), ${ }^{4}$ the evidence pertaining specifically to the impact of maternal depression on infant survival is limited and inconclusive.

In a Taiwanese national registry linkage study, maternal depression in the year after birth was independently associated with a subsequent 1.5-fold (95\% CI 1.2 to 1.9) of increased mortality risk for children up to 5 years of age. ${ }^{5}$ In an Ethiopian cohort study, ${ }^{6}$ the 561 children of women who had been screened for depression in the previous year, and who were born within 1 year of their mother's assessment, were followed up 
for mortality until the age of 3 years; there was a borderline association between antenatal maternal depression and child mortality (rate ratio (RR) $2.3,95 \%$ CI 1.0 to 4.9 ), and a greater mortality risk when combined with mothers' experiences of physical (RR 4.0, 95\% CI 1.6 to 10.1 ) or emotional (RR 3.7, 95\% CI 1.3 to 10.4) abuse. In a recent cohort study from Ethiopia, ${ }^{7}$ of the 969 children whose mothers were screened for common mental disorder (CMD) at pregnancy and after birth, and who were followed up for 3.5 years, there was no association between: antenatal CMD, postnatal CMD or both, with child mortality. Sudden infant death syndrome is the leading cause of infant deaths between 1 month and 1 year in developing countries, ${ }^{8}$ and probable postnatal depression has been identified as one of the risk factors in developed country settings. ${ }^{9}$ No studies to date appear to have assessed the impact of depression in the immediate postnatal period on subsequent infant mortality. This is the period of maximum vulnerability for child deaths, ${ }^{10}$ and a critical time for mother-child bonding, ${ }^{11}$ when the demands of providing good quality nurturance and care are most challenging for the mother.

In this paper, we present findings on the association between probable postnatal depression, and subsequent infant mortality and morbidity from the Depression nested within the ObaapaVitA and Newhints trials (DON) study carried out to assess the burden, determinants and consequences of antenatal and postnatal depression in rural Ghana.

\section{MATERIALS AND METHODS}

\section{Study design}

DON is a cohort study of perinatal Depression nested within the ObaapaVitA ${ }^{12}$ and Newhints ${ }^{13}$ cluster randomised controlled trials conducted in seven contiguous districts in the Brong-Ahafo Region of Ghana. The ObaapaVitA trial evaluated the effect of weekly vitamin A supplementation in women of reproductive age on maternal mortality, and the Newhints trial assessed the impact of home visits by lay community health volunteers on neonatal mortality. These trials were supported by 4 weekly home surveillance of women of reproductive age to identify pregnancies, births, infant and maternal deaths and morbidity information. DON was carried out from late January 2008 to early August 2009, and comprised depression assessments in the 4 weekly surveillance visits following identification of pregnancy and in the visits following reporting of a delivery.

Both trials were registered with clinicaltrials.gov: ObaapaVitA, number NCT00211341; Newhints, number NCT00623337.

\section{Setting}

The study area covers a population of about $700000^{14}$ with more than 120000 women of reproductive age, ${ }^{13}$ and more than 15000 births a year. The infant mortality rate (IMR) is 63/1000 child-years and the neonatal mortality rate is $31 / 1000$ live births. ${ }^{12}$ The area is predominantly rural, but has four medium-sized towns (populations of at least 40000 ). Routine identification and treatment for antenatal/postnatal depression in primary care does not exist. Access to orthodox mental health services is limited, and help for mental ill health is generally provided by traditional healers and spiritual/ healing churches. ${ }^{15}$

\section{Participants}

Participants were mothers who had a live birth reported between 24 March 2008 and 11 July 2009, who were screened for probable postnatal depression between 4 and 12 weeks post partum (some of whom had also had depression assessed at pregnancy), and whose infants survived to this point.

\section{Data collection}

Data were collected through the surveillance system supporting the ObaapaVitA and Newhints trials, in which all women of reproductive age were visited every 4 weeks by resident fieldworkers, who collected data on pregnancies, births and deaths. The sociodemographic, socioeconomic, pregnancy and obstetric history data were collected when a pregnancy was identified, and a pregnancy depression assessment was conducted at the next 4 weekly visit. Information about the pregnancy, delivery, the baby (or babies) and the newborn care practices was collected at the first visit after the birth. A postnatal assessment was carried out at the following 4 weekly visit. Subsequent 4 weekly visits were made to collect data on the infant until their first birthday.

\section{Exposure}

The assessments of antenatal and postnatal depression were made by administering the Twi (widely spoken language in Ghana and the study area) version of the nineitem Patient Health Questionnaire (PHQ-9). The PHQ-9 is a structured questionnaire that enquires after the nine symptom-based criteria for a probable diagnosis of Diagnostic and Statistical Manual of Mental Disorders, fourth edition (DSM-IV) ${ }^{16}$ depression, duration and severity of the symptoms. Symptoms reported as present for at least half the time in the previous 2 weeks are rated positively. Either depression or anhedonia (loss of interest or pleasure) must be rated, with a total of five or more symptoms for major depression and two to four symptoms for minor depression. In contrast with symptom-based scale scores, these criteria therefore identify individuals with persistent and pervasive symptoms, characteristic of a clinically significant depressive episode. In its initial review, it recorded sensitivity and specificity of 0.88 at a cut-off of $10,{ }^{16}$ and high positive predictive value. ${ }^{17}$ The PHQ-9 has been previously validated among women who recently delivered within the same study population and showed superior psychometric properties when compared with the Edinburgh Postnatal Depression Scale ${ }^{18}$-it recorded a sensitivity of 
0.94 and specificity of 0.75 at a cut-off of 5 . As part of the cross-cultural adaptation of the PHQ-9 to the study setting referred to above, qualitative accounts supported the construct of depression that existed in this setting, as mothers referred to the experience as a 'worrying' or 'thinking' sickness.

\section{Outcomes}

Outcomes were assessed every 4 weeks by resident fieldworkers until 1 year after birth and, in one of these visits, a postnatal depression assessment was also completed. The primary outcome was all-cause early infant mortality expressed per 1000 infant-months of follow-up from the time of postnatal assessment to 6 months of age. The secondary outcomes were (1) all-cause infant mortality from the time of postnatal assessment to 12 months of age and (2) reported infant morbidity from the time of the postnatal DON assessment to 12 months of age. Morbidity indicators were: any ill health on the day of visit; any serious illness in the past month requiring care-seeking outside the home; and occurrence in the past $24 \mathrm{~h}$ of diarrhoea, vomiting, cough, fever and frequent crying.

\section{Potential confounders}

A priori potential confounders were: maternal characteristics (age, marital status, education status, occupation, ethnicity, religion and rural or urban residence); pregnancy and obstetric variables (parity, preterm delivery and mode of delivery); and infant characteristics (sex and perceived size of baby as a proxy for birth weight). In addition, an overall socioeconomic 'score' for each woman was generated using factor analysis techniques after the methods described by Vyas and Kumaranayake. ${ }^{19}$ Briefly, this score is based on household possession/absence of a set of assets grouped under several themes including ownership of land, animals, presence of electricity and/or electrical goods, water source, type of latrine, number of residents per room and materials used in construction of housing. Principal component analysis (using the correlation matrix to ensure that all included variables had equal weight) was then performed to assign each asset a factor score based on the strength of its correlation to the first principle component (which is assumed to be the optimal measure of economic status). Individual asset factor scores were summed for each woman to provide a measure of her overall socioeconomic score, where the higher the score, the higher the assumed economic status of the household. Women were ranked according to these socioeconomic scores into wealth quintile groups. Intervention status was not included as prevalence of postnatal depression was comparable in the intervention and control arms of the Newhints trial.

\section{Statistical methods}

Multiple births were excluded from all analyses given the high risk associated with infant mortality, particularly in Africa, ${ }^{20}$ and the possible association with probable postnatal depression. ${ }^{21}$ Poisson regression was used to examine the association between probable postnatal depression and infant mortality, adjusting for the a priori potential confounders listed above. In addition, we tested for the moderating effect of relevant covariates by fitting appropriate interaction terms in the model. In order to account for the possible influence of reverse causality from illness that culminated in the deaths that occurred close to ascertainment of probable postnatal depression status, we conducted sensitivity analyses excluding those deaths that occurred, first within 1 week and second within 30 days after postnatal depression assessment. Only those babies whose deaths were ascertained within the period of follow-up were included in the analyses.

Additional analyses compared infant mortality between: women not depressed at either antenatal or postnatal assessments, women depressed only at antenatal assessment, women depressed only at postnatal assessment and those depressed at both. Kaplan-Meier survivor function was used to plot cumulative infant survival graphs for the four groups from birth until 12 months of age, excluding any deaths that occurred before the postnatal depression assessment.

For the association between probable postnatal depression and infant morbidity, we used mixed-effects repeated measures logistic regression models with random intercept at the infant level, including the same potential confounders aforementioned, plus month of visit. The delta method was applied to predict risk/rate ratios (RR) with 95\% CIs for individuals with zero random effect using the marginal standardisation technique. ${ }^{22}$ We also estimated the attributable risk per cent (percentage of early infant deaths among women with probable postnatal depression, ie, attributed to exposure to probable postnatal depression) and the population attributable fraction (percentage of early infant deaths that would be prevented if the effect of probable postnatal depression is removed). All analyses were conducted using STATA V.11. ${ }^{23}$

\section{Ethical considerations}

Informed consent (by signature or thumbprint) was obtained for each woman.

\section{RESULTS}

Figure 1 summarises the recruitment profile. 21283 deliveries were identified through 4 weekly surveillance visits between 24 March 2008 and 11 July 2009. Of these, 18356 mothers (86\%) completed the PHQ-9 depression screen after birth (live/stillbirth) between 4 and 12 weeks; $1450(7 \%)$ were absent at the screening visit, 208 (1\%) women were visited but PHQ-9 was not completed, $1244(5.8 \%)$ were screened but not within 4 and 12 weeks and $25(0.1 \%)$ declined to participate. After excluding stillbirths, multiple births, infant deaths that 
occurred before postnatal depression assessment and mothers who died after postnatal depression assessment, 17032 were eligible for analyses. Of these, 16560 (97\%) mother-infant pairs had complete information on all potential confounders, contributing 130 infant deaths and 67457.4 infant-months of follow-up to the analysis. Of these, 12618 had also been screened for depression during pregnancy.

The prevalence of DSM-IV postnatal depression was $3.5 \%(\mathrm{n}=591 ; 95 \%$ CI 3.2 to 3.7$)$. A detailed profile of study participants is provided in a companion paper. ${ }^{24}$ In brief, the population was predominantly rural $(70 \%)$, $90 \%$ percent of the mothers were married or cohabiting and $36 \%$ had no formal education.

\section{Postnatal depression and risk of infant death}

All-cause infant mortality from the time of postnatal depression assessment up to 6 months of age (adjusted RR 2.86, 95\% CI 1.58 to 5.19) was almost three times higher among infants of depressed mothers compared to those not depressed, and almost twofold up to 12 months of age (adjusted RR 1.88, 95\% CI 1.09 to 3.24; table 1). The effect at 6 months was similar when infant deaths occurring within 7 days or within 30 days of the date of the postnatal depression assessment were excluded (table 1). Among the potential confounders included in the model only timing of delivery (preterm) was also associated with an almost fivefold increased risk of infant deaths up to 6 months of age (adjusted RR 4.61, 95\% CI 2.02 to 10.51). A further test for effect modification suggested the associations observed with probable postnatal depression and early delivery with infant deaths were independent, as there was no evidence of interaction. We further estimate that $65 \%$ of early infant deaths among depressed mothers are attributable to their depression. This translates to a population attributable fraction of $8.8 \%$ of all early infant deaths in this population, which has a relatively low prevalence of probable DSM-IV major/minor postnatal depression of $3.5 \%$.
Figure 1 Flow chart on analysis cohort (PHQ-9, nine-item Patient Health Questionnaire).

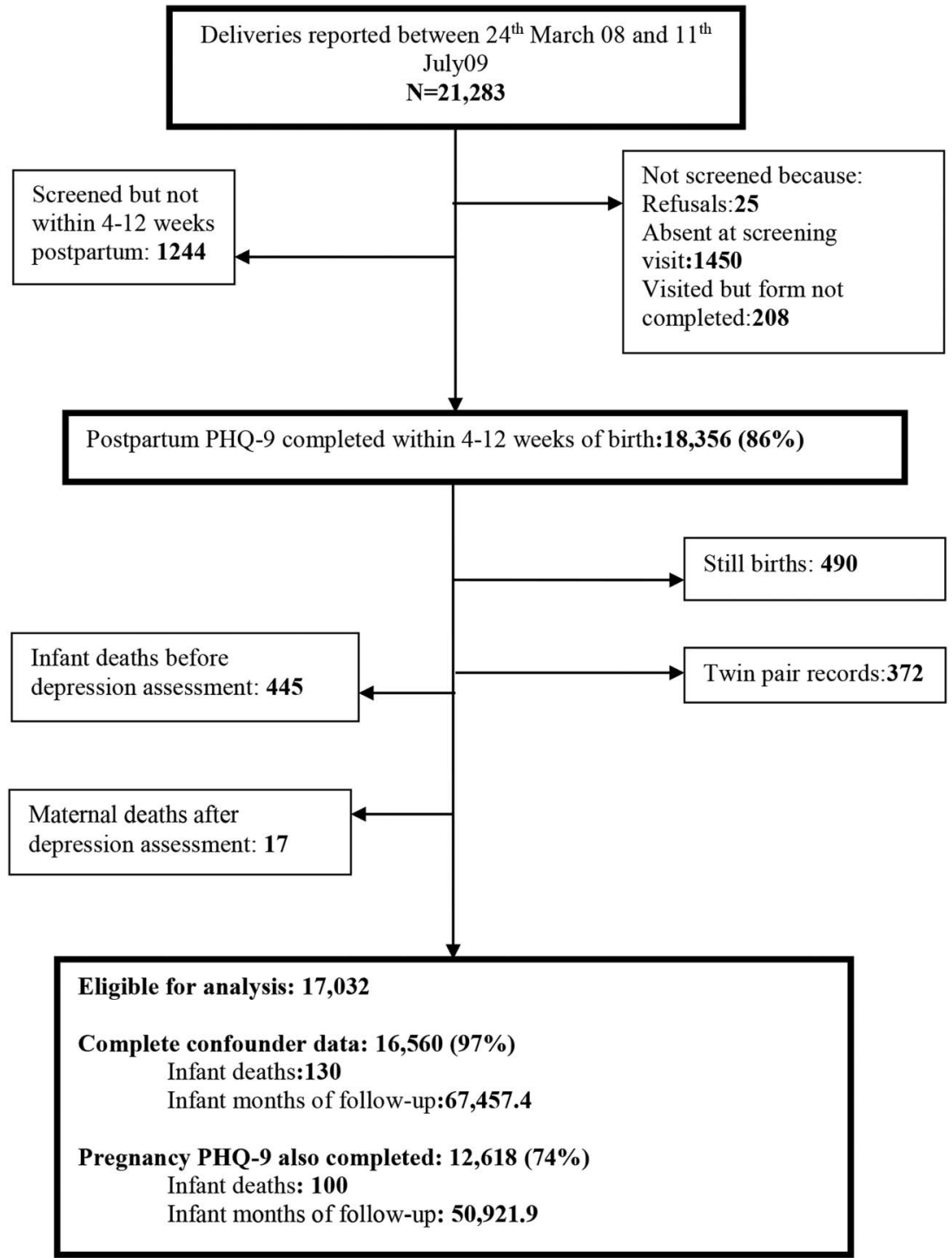




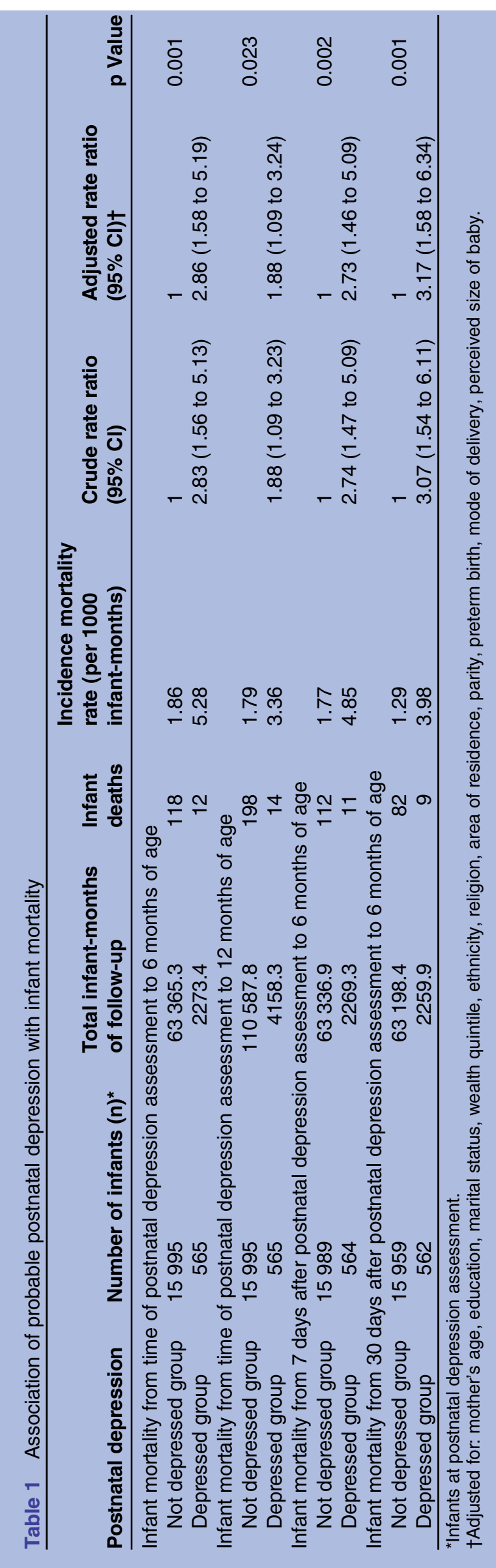

Figure 2 shows the infant survival curves up to 12 months of age excluding babies who died before the time of the postnatal depression assessment, for the four groups categorised by maternal depression status. As can be seen, survival is lowest for infants of mothers with probable postnatal depression (whether postnatal only, or antenatal and postnatal) compared to those with no depression or those with only probable antenatal depression. This is confirmed by Poisson regression analysis (see online supplementary table 1.1), which showed that infant mortality among offspring of mothers depressed antenatally but not postnatally was similar to that of offspring of mothers who were not depressed at either assessment point (adjusted RR 1.32, 95\% CI 0.68 to 2.56 $\mathrm{p}=0.408$ ). In contrast, infant mortality was substantially higher for offspring of mothers with probable postnatal depression, whether they also had probable antenatal depression or not (adjusted RR 3.19, 95\% CI 0.99 to $10.17 \mathrm{p}=0.050)$. Although the prevalence of exposure was comparable between arms and there was no statistically significant effect of the Newhints intervention on neonatal mortality, we conducted a post hoc analysis adjusting for the intervention effect of Newhints and the results were unchanged, as expected (adjusted RR 2.82, $95 \%$ CI 1.55 to $5.14 \mathrm{p}=0.001$ ).

\section{Risk of infant morbidity}

Table 2 shows increased risks associated with probable postnatal depression for infant morbidity indicators. Additional analysis indicated that these increased risks of infant morbidity were also apparent for women with antenatal depression only, although with generally smaller effect sizes than those for either postnatal depression only, or for those with both antenatal and postnatal depression (see online supplementary table 1.2).

\section{DISCUSSION}

We found that maternal probable postnatal depression was independently associated with subsequent increased risk of infant mortality up to 6 months and up to 12 months of age and with increased infant morbidity. Analysis of the separate effects of probable antenatal and postnatal depression suggests that the postnatal period may be critical for infant survival; the offspring of mothers with probable postnatal depression have an increased mortality risk, while those with probable antenatal depression have an increased morbidity risk. Thus, probable antenatal depression leads to more morbidity than mortality, and this is confirmed in our companion paper on consequences of antenatal depression for the mother and newborn.

Our results are consistent with recent findings from a large national register linkage study in Taiwan. ${ }^{5}$ However, our study is unique in that it focuses on depression over the immediate postnatal period, and on infant mortality 
Figure 2 Child survival by presence of probable antenatal and/or postnatal depression up to 6 months of age, restricted to babies alive at the time of the postnatal depression assessment.

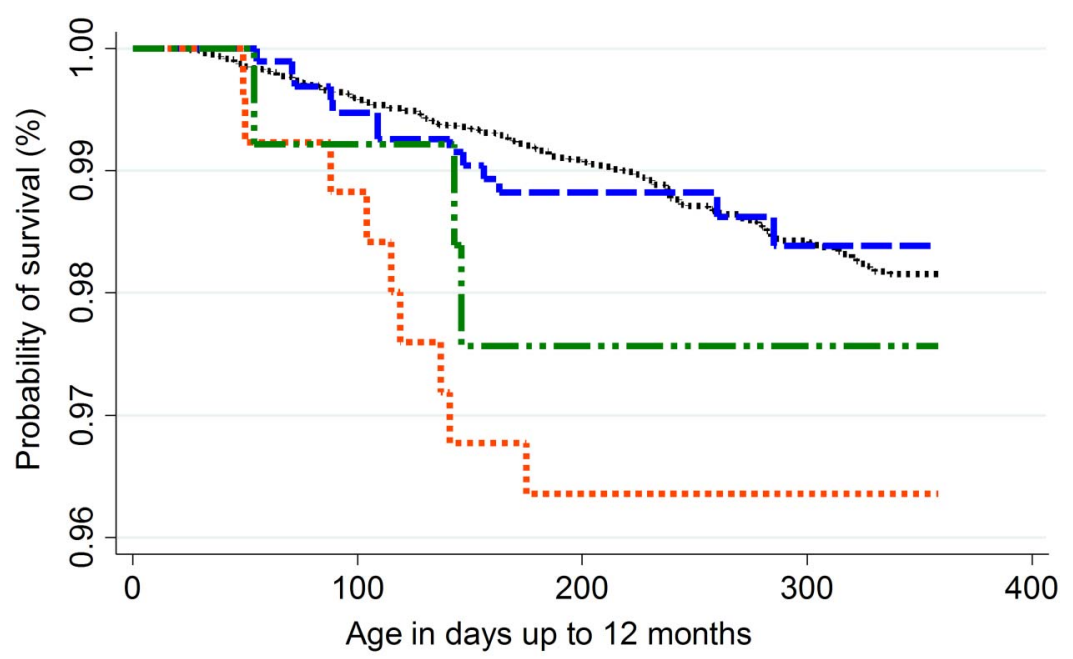

,.,.,., Neither antenatal nor postnatal depression - Antenatal depression only

Postnatal depression only in the first 6 months of life. Other strengths are that: we employed a large prospective cohort, measured depression exposure using a validated and standardised tool, and encountered few refusals.

We acknowledge several limitations: despite the prospective design we cannot exclude respondent bias, particularly an effect of maternal depression on the mothers' reports of infant morbidity. We also acknowledge the challenges in establishing causality, given the often chronic, remitting and relapsing nature of depression. The PHQ-9, however, can only provide approximations to DSM-IV depression, it is not a diagnostic tool and we are only able to provide probable diagnosis of postnatal depression in our study. We also note that our nested design meant that women had regular 4 weekly visits from resident fieldworkers enquiring about their health; these visits may have some therapeutic effects. These therapeutic effects may explain our relatively low prevalence (compared to previous high estimates from sub-Saharan Africa), and thus suggest that the prevalence of probable postnatal depression may have been underestimated in our setting. Furthermore, even though we set out to investigate associations between probable postnatal depression and all-cause infant mortality, it would have been useful to assess cause-specific mortality in order to further understand the mechanisms that explain why risk is greater among mothers with postnatal depression. Unfortunately, we could not obtain these data at the time of conducting these analyses. We may conjecture, however, that the excess mortality risk observed may be attributed to the increased morbidity risk among infants whose mothers are depressed. For example, dehydration from infant diarrhoea is a wellestablished cause of infant death. ${ }^{25}$ Finally, our results may only be generalisable to singletons, given the fact that we excluded multiple births in our analyses.

In this study, we sought to identify probable clinically significant depressive episodes, and therefore used the
PHQ-9 item ratings to give an approximation to DSM-IV major or minor depression diagnostic categories. ${ }^{1726}$ As expected, this gave a lower prevalence than using the PHQ-9 CMD cut-off 5+ score (3.5\% compared to $6.7 \%)$. The only systematic review of prevalence rates of postnatal depression based mainly on score cut-offs in sub-Saharan Africa reported much higher estimates ( $18.3 \%(95 \%$ CI 17.9 to $19.1 \%)),{ }^{27}$ as expected; prevalence estimates from primary studies ranged from a low of $3.2 \%$ to a high of $48 \%$. The number of infant deaths reported in this analysis is relatively low for such a reasonably large sample and the known IMR in this setting. The reason for this is that though the sample comprised all deliveries recorded between 2008 and 2009, we only included infant deaths that we had information on during the period of follow-up specified, and those whose mothers had a depression assessment completed. It is thus very possible that some infant deaths occurred post the follow-up period, but these were not counted as we did not follow-up all babies right up to 6 and 12 months of age. We also think that the timing of assessments may explain the relatively fewer number of deaths in our cohort; the contribution to the IMR could have been lost for mothers assessed later.

Our report has raised important dimensions on the risk factors for early infant deaths by confirming the already established evidence of the association between poor birth outcomes, and neonatal and infant mortality, and producing new evidence of the potential role of poor maternal mental health on child survival. There are several possible reasons to explain why probable postnatal depression may be associated with infant deaths. First, we cannot rule out reverse causality in this study. Thus, a mother's depression may have been triggered by her infant's severe or terminal illness, and the subsequent death of the infant may not be confidently attributed to depression. Reassuringly, sensitivity analyses excluding infant deaths that occurred 1 week, or 4 weeks 
Table 2 Association between maternal probable postnatal depression and infant morbidity

\begin{tabular}{|c|c|c|c|c|c|c|}
\hline Outcome & Number of infants* & Number of visits (n) & Percentage of visits $\dagger$ & Crude risk ratio $(95 \% \mathrm{Cl})$ & Adjusted risk ratio $(95 \% \mathrm{Cl}) \ddagger$ & p Value \\
\hline \multicolumn{7}{|l|}{ Any ill health on day of visit } \\
\hline Depressed group & 564 & 4101 & 21.5 & 1.90 (1.69 to 2.10$)$ & $1.70(1.53$ to 1.88$)$ & $<0.001$ \\
\hline \multicolumn{7}{|c|}{ Any serious illness in past month requiring care seeking } \\
\hline Depressed group & 564 & 4101 & 14.6 & $1.35(1.20$ to 1.50$)$ & 1.34 (1.19 to 1.49$)$ & $<0.001$ \\
\hline \multicolumn{7}{|c|}{ Diarrhoea within past $24 \mathrm{~h} \S$} \\
\hline Not depressed group & 9875 & 47877 & 6.4 & 1 & 1 & \multirow[t]{2}{*}{$<0.001$} \\
\hline Depressed group & 391 & 1991 & 12.1 & 2.17 (1.73 to 2.60$)$ & $1.80(1.45$ to 2.14$)$ & \\
\hline Depressed group & 304 & 304 & 9.9 & 2.15 (1.39 to 2.91 ) & $1.98(1.26$ to 2.71$)$ & 0.007 \\
\hline \multicolumn{7}{|l|}{ Fever within past $24 \mathrm{~h} \S$} \\
\hline Not depressed group & 9875 & 47878 & 8.5 & 1 & 1 & \multirow[t]{2}{*}{$<0.001$} \\
\hline Depressed group & 391 & 1991 & 15.0 & 2.10 (1.72 to 2.47 ) & $1.80(1.49$ to 2.11$)$ & \\
\hline \multicolumn{7}{|l|}{ Cough within past $24 \mathrm{~h} \S$} \\
\hline Not depressed group & 9875 & 47877 & 14.0 & 1 & 1 & \multirow{2}{*}{$<0.001$} \\
\hline Depressed group & 391 & 1991 & 20.2 & $1.60(1.36$ to 1.84$)$ & $1.49(1.28$ to 1.70$)$ & \\
\hline
\end{tabular}


after the depression assessment, showed similar findings as shown in table 1 . Nevertheless, causality can be convincingly demonstrated by a trial showing that treating postnatal depression results in improved infant survival.

Second, the strong and independent associations with morbidity observed in this report and by other authors in Africa, ${ }^{28}$ South Asia ${ }^{29}{ }^{30}$ and high-income countries, ${ }^{31} 32$ provide evidence of the biological plausibility to our findings. Third, the following potential mechanisms could explain the association observed: poor mother-infant interaction, ${ }^{33}$ reduced stimulation, ${ }^{34}$ inability to prevent injuries, ${ }^{35} 36$ and poor adherence to child survival interventions ${ }^{29} 37$ including childhood immunisations, ${ }^{30}$ good hygiene behaviours such as handwashing before feeding, and safe food preparation and storage, ${ }^{38}$ and prompt care seeking for childhood illnesses. ${ }^{1}$ It is interesting to note that in this study, within the context of perinatal depression, probable postnatal depression alone is the main determinant of infant deaths in the immediate postpartum period, and not when probable postnatal depression is combined with probable antenatal depression. The possible reasons for this are that: (1) the potential mechanisms outlined above are more relevant in the postnatal period; (2) in this setting up to $85 \%$ of antenatal depression remits spontaneously, ${ }^{24}$ and this may suggest a gradual weakening effect of probable antenatal depression by the time of birth. Probable antenatal depression may not be strongly associated with mortality outcomes but it is certainly implicated as a risk factor for infant morbidity. The pathway linking probable antenatal depression and infant morbidity may be explained by downstream effects of reported consequences of antenatal depression such as: suboptimal care provided during the neonatal period, ${ }^{39}$ poor maternal self-care, nutrition, lack of sleep ${ }^{40}$ and peripartum/postpartum complications experienced by the mother. ${ }^{41}$

Our study is the first large population-based cohort from sub-Saharan Africa to investigate the association between probable postnatal depression and infant survival, supported by a plausible mechanism indicated by associations with infant illness. We have reported robust findings of public health importance, but would encourage further testing of this hypothesis in other settings to improve the external validity of our findings. In the meantime, there is a strong case to intervene in cases of mothers who are depressed. Aside from the effect on infants, in other studies, the disorder has been shown to be associated with considerable disability and reduced quality of life for the mother, ${ }^{42}$ and to affect the family. ${ }^{43}$ Our findings provide support for the detection and treatment of clinically significant depression in the postnatal period, and re-echoes the call to address maternal depression as a human rights issue needing urgent attention. ${ }^{44}$

Evidence for the successful integration of mental health in primary care using lay community human resources in low-income and middle-income settings exists, ${ }^{145-47}$ and models for scale up have been provided by the WHO through its Mental Health Gap Action Programme (mhGAP) ${ }^{48}$ The mhGAP primary care guidelines on tackling perinatal depression require that antidepressants should be avoided as far as practicable, but recommend, as a first step, the need to address psychosocial stressors, and to reactivate social networks through psychological and psychosocial interventions such as interpersonal psychotherapy, behavioural activation, or cognitive-behavioural therapy, in addition to effective referral and supervision support from specialists.

We recommend that trials are urgently designed to evaluate the impact of different strategies to tackle postnatal depression. We further recommend that these trials should include infant mortality as an outcome to clarify whether the association found is causal, and to test whether treating postnatal depression in conjunction with improving the care-giving environment, as suggested by other authors, ${ }^{49}$ particularly for babies born preterm, leads to improved infant survival. Tackling postnatal depression alongside improving pregnancy outcomes may, therefore, have a role to play in achieving the millennium development goal for child survival as well as being essential for optimal child development.

\section{Author affiliations}

${ }^{1}$ Kintampo Health Research Centre, Ghana Health Service, Kintampo, Ghana ${ }^{2}$ Faculty of Epidemiology and Population Health, London School of Hygiene and Tropical Medicine, London, UK

${ }^{3}$ Department of Public Health, Academic Medical Centre, Amsterdam, The Netherlands

${ }^{4}$ Health Services and Population Research Department, Institute of Psychiatry, King's College London, London, UK

Acknowledgements The authors would like to thank the women who were part of this study for consenting and for their time. The surveillance team who collected and supervised the collection of the data are duly commended for their hard work. They also thank the staff of the Kintampo Health Research Centre, and especially those in the mental health unit, for their role in training field staff. They are also very grateful to the Ghana Health Service institutions within the KHRC study area for maternal and child health research collaborations.

Contributors BW, MP and BRK designed the study.BW conducted all analysis and wrote the paper with input from MP and BRK. BW, GTA, SS, SA-E and SO-A conducted the study and conducted a critical review of the manuscript. SD, SS and LG managed the data, conducted a critical review of the manuscript and conducted some of the analyses. All the authors read and approved the final manuscript.

Funding This study was provided funding by the UK Department for International Development (EPHIHD66), Save the Children's 'Saving Newborn Lives' programme from The Bill \& Melinda Gates Foundation (EPIDVA37) and WHO (EPNPVE28).WB also received support for his fees, travel and subsistence, from the Psychiatric Research Trust (PALTHPA), London School of Hygiene and Tropical Medicine, and the UK Department for International Development.

Competing interests None declared.

Ethics approval Ethical approval for the DON study was granted by the ethics committees of the Kintampo Health Research Centre and the London School of Hygiene and Tropical Medicine.

Provenance and peer review Not commissioned; externally peer reviewed.

Data sharing statement Additional data can be accessed via the Dryad data repository at http://datadryad.org/ with the doi:10.5061/dryad.4tj59. 
Open Access This is an Open Access article distributed in accordance with the Creative Commons Attribution Non Commercial (CC BY-NC 4.0) license, which permits others to distribute, remix, adapt, build upon this work noncommercially, and license their derivative works on different terms, provided the original work is properly cited and the use is non-commercial. See: http:// creativecommons.org/licenses/by-nc/4.0/

\section{REFERENCES}

1. Prince M, Patel V, Saxena S, et al. No health without mental health. Lancet 2007;370:859-77.

2. Rahman A, Prince M. Mental health in the tropics. Ann Trop Med Parasitol 2009;103:95-110.

3. Walker SP, Wachs TD, Gardner JM, et al. Child development: risk factors for adverse outcomes in developing countries. Lancet 2007;369:145-57.

4. Webb R, Abel K, Pickles A, et al. Mortality in offspring of parents with psychotic disorders: a critical review and meta-analysis. Am J Psychiatry 2005;162:1045-56.

5. Chen YH, Tsai SY, Lin HC. Increased mortality risk among offspring of mothers with postnatal depression: a nationwide population-based study in Taiwan. Psychol Med 2011;41:2287-96.

6. Deyessa N, Berhane Y, Emmelin M, et al. Joint effect of maternal depression and intimate partner violence on increased risk of child death in rural Ethiopia. Arch Dis Child 2010;95:771-5.

7. Adewuya AO, Hanlon C, Medhin G, et al. Perinatal common mental disorders and child survival in Ethiopia. J Paediatr Child Health 2014;50:57-63.

8. Moon RY, Horne RS, Hauck FR. Sudden infant death syndrome. Lancet 2007;370:1578-87.

9. Sanderson CA, Cowden B, Hall DM, et al. Is postnatal depression a risk factor for sudden infant death? Br J Gen Pract 2002;52:636-40.

10. Rajaratnam JK, Marcus JR, Flaxman AD, et al. Neonatal, postneonatal, childhood, and under-5 mortality for 187 countries, 1970-2010: a systematic analysis of progress towards Millennium Development Goal 4. Lancet 2010;375:1988-2008.

11. Egeland B, Farber EA. Infant-mother attachment: factors related to its development and changes over time. Child Dev 1984;55:753-71.

12. Kirkwood BR, Hurt L, Amenga-Etego S, et al. Effect of vitamin A supplementation in women of reproductive age on maternal survival in Ghana (ObaapaVitA): a cluster-randomised, placebo-controlled trial. Lancet 2010;375:1640-9.

13. Kirkwood BR, Manu A, Tawiah-Agyemang C, et al. NEWHINTS cluster randomised trial to evaluate the impact on neonatal mortality in rural Ghana of routine home visits to provide a package of essential newborn care interventions in the third trimester of pregnancy and the first week of life: trial protocol. Trials 2010;11:58

14. GHS. Ghana Health Service. Ghana Health Service-Brong Ahafo Region: Population by districts: Ghana Health Service; 2011. [cited 2012 26.05.2012]. http://www.ghanahealthservice.org/region.php? $\mathrm{dd}=6$ \& region=Brong\&nbsp;Ahafo\&nbsp;Region

15. Ae-Ngibise K, Cooper S, Adiibokah E, et al. 'Whether you like it or not people with mental problems are going to go to them': a qualitative exploration into the widespread use of traditional and faith healers in the provision of mental health care in Ghana. Int Rev Psychiatry 2010;22:558-67.

16. Kroenke K, Spitzer RL, Williams JBW. The PHQ-9: validity of a brief depression severity measure. J Gen Intern Med 2001;16:606-13.

17. Kroenke KS, Spitzer RL. The PHQ-9: a new depression diagnostic and severity measure. Psychiatr Ann 2002;32:91-7.

18. Weobong B, Akpalu B, Doku V, et al. The comparative validity of screening scales for postnatal common mental disorder in Kintampo, Ghana. J Affect Disord 2009;113:109-17.

19. Vyas S, Kumaranayake L. Constructing socio-economic status indices: how to use principal components analysis. Health Policy Plan 2006;21:459-68.

20. Zwane E. Socioeconomic and maternal determinants of infant mortality: an analysis using the Swaziland demographic health survey 2007. Internet J Epidemiol 2012;10:2.

21. Choi Y, Bishai D, Minkovitz CS. Multiple births are a risk factor for postpartum maternal depressive symptoms. Pediatrics 2009;123:1147-54.

22. Localio AR, Margolis DJ, Berlin JA. Relative risks and confidence intervals were easily computed indirectly from multivariable logistic regression. J Clin Epidemiol 2007;60:874-82.

23. STATA. Statistics/data analysis. 11 edn. Texas: Stata Press, 2009

24. Weobong B, Ten Asbroek AH, Soremekun S, et al. Determinants of postnatal depression in Rural Ghana: findings from the Don population based cohort study. Depress Anxiety 2015;32: $108-19$.
25. WHO. Diarrhoeal Disease: Fact sheet N 330. Geneva: WHO, 2013.

26. Martin A, Rief W, Klaiberg A, et al. Validity of the Brief Patient Health Questionnaire Mood Scale (PHQ-9) in the general population. Gen Hosp Psychiatry 2006;28:71-7.

27. Sawyer A, Ayers S, Smith H. Pre- and postnatal psychological wellbeing in Africa: a systematic review. J Affect Disord 2010;123:17-29.

28. Ross J, Hanlon C, Medhin G, et al. Perinatal mental distress and infant morbidity in Ethiopia: a cohort study. Arch Dis Child Fetal Neonatal Ed 2011;96:F59-64.

29. Rahman A, lqbal Z, Bunn J, et al. Impact of maternal depression on infant nutritional status and illness: a cohort study. Arch Gen Psychiatry 2004;61:946-52

30. Rahman A, Bunn J, Lovel H, et al. Maternal depression increases infant risk of diarrhoeal illness: a cohort study. Arch Dis Child 2007;92:24-8

31. Ban L, Gibson J, West J, et al. Association between perinatal depression in mothers and the risk of childhood infections in offspring: a population-based cohort study. BMC Public Health 2010;10:799.

32. Groer MW, Morgan K. Immune, health and endocrine characteristics of depressed postpartum mothers. Psychoneuroendocrinology 2007;32:133-9.

33. Cooper P, Tomlinson M, Swartz L, et al. Post-partum depression and the mother-infant relationship in a South African peri-urban settlement. Br J Psychiatry 1999;175:554-8.

34. Baker-Henningham $\mathrm{H}$, Powell $\mathrm{C}$, Walker $\mathrm{S}$, et al. Mothers of undernourished Jamaican children have poorer psychosocial functioning and this is associated with stimulation provided in the home. Eur J Clin Nutr 2003;57:786-92.

35. Howe LD, Huttly SR, Abramsky T. Risk factors for injuries in young children in four developing countries: the Young Lives Study. Trop Med Int Health 2006;11:1557-66.

36. O'Connor TG, Davies L, Dunn J, et al. Distribution of accidents, injuries, and illnesses by family type. ALSPAC Study Team. Avon Longitudinal Study of Pregnancy and Childhood. Pediatrics 2000;106:E68.

37. Patel V, DeSouza N, Rodrigues M. Postnatal depression and infant growth and development in low income countries: a cohort study from Goa, India. Arch Dis Child 2003;88:34-7.

38. Thongkrajai E, Thongkrajai P, Stoeckel J, et al. Socioeconomic and health programme effects upon the behavioral management of diarrhoeal disease in northeast Thailand. Asia Pac J Public Health 1990;4:45-52.

39. Hanlon C, Medhin G, Alem A, et al. Impact of antenatal common mental disorders upon perinatal outcomes in Ethiopia: the P-MaMiE population-based cohort study. Trop Med Int Health 2009:14:156-66.

40. Weissman MM, Olfson M. Depression in women: implications for health care research. Science 1995;269:799-801.

41. Weobong B, Ten Asbroek AH, Soremekun S, et al. Association of antenatal depression with adverse consequences for the Mother and Newborn in Rural Ghana: findings from the DON population-based cohort study. PLOS ONE 2014;9:e116333.

42. Nicholson WK, Setse R, Hill-Briggs F, et al. Depressive symptoms and health-related quality of life in early pregnancy. Obstet Gynecol 2006;107:798-806.

43. O'Hara MW. Postpartum depression: causes and consequences. New York: Springer-Verlag, 1995.

44. Wachs TD, Black MM, Engle PL. Maternal depression: a global threat to children's health, development, and behavior and to human rights. Child Dev Perspect 2009;3:51-9.

45. Patel V, Weiss HA, Chowdhary N, et al. Effectiveness of an intervention led by lay health counsellors for depressive and anxiety disorders in primary care in Goa, India (MANAS): a cluster randomised controlled trial. Lancet 2010;376:2086-95.

46. Araya $\mathrm{R}$, Rojas $\mathrm{G}$, Fritsch $\mathrm{R}$, et al. Treating depression in primary care in low-income women in Santiago, Chile: a randomised controlled trial. Lancet 2003;361:995-1000.

47. Rahman A, Malik A, Sikander S, et al. Cognitive behaviour therapy-based intervention by community health workers for mothers with depression and their infants in rural Pakistan: a cluster-randomised controlled trial. Lancet 2008;372: 902-9.

48. Dua T, Barbui C, Clark N, et al. Evidence-based guidelines for mental, neurological, and substance use disorders in low- and middle-income countries: summary of WHO recommendations. PLoS Med 2011;8:e1001122.

49. Nylen KJ, Moran TE, Franklin CL, et al. Maternal depression: a review of relevant treatment approaches for mothers and infants. Infant Mental Health J 2006;27:327-43. 